\section{The sensing eye: Intimate vision in couple dancing}

\section{Maria Törnqvist (1) and Tora Holmberg}

Department of Sociology, Uppsala University, Sweden
Ethnography

202I, Vol. 0(0) I-I7

(C) The Author(s) 2021

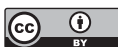

Article reuse guidelines: sagepub.com/journals-permissions DOI: $10.1177 / 14661381211038430$ journals.sagepub.com/home/eth @SAGE

\begin{abstract}
Vision tends to be associated with the mind and theorized as the least bodily sense. Notably, the eye often symbolizes distance. Foregrounding the fleshy embeddedness of the gaze through an analysis of jitterbug, the present article stresses the bodily and intimate significance of vision in dancing. Analyzing ethnographic observation and interview data, we develop a phenomenological approach to dance and gaze that stresses the need to address multi-sensoriality, adding vision to the perspective of tactilekinesthetic touch. However, the article develops a more-than-optic perspective on "dancing as vision." The "sensing eye" is analyzed, first, as a body technique central to couple dancing and, second, as the use and meaning of vision across spatial distance, as between dancers and bystanders. Throughout the article, the dance floor unveils connections between eye and body, self and other, and passive and active and thus pushes notions of emplacement and embodiment.
\end{abstract}

\title{
Keywords
}

dance, body, vision, ethnography, phenomenology, popular culture, embodiment

\section{Introduction}

Dance is certainly many things: a rhythmic, energy-producing experience (Crespi, 2014), a ritualistic confirmation of belonging (Lefevre, 2014), and a practice of sensuous mobility through which subjects and places come alive (Atkinson and Duffy, 2019). In addition, and perhaps more importantly, dancing is about social relations and involves a delicate drama of closeness regulated by the symbolic borders played out on and by the dancing bodies (Törnqvist, 2013). Dance intimacy is often explored from the perspective of touching bodies in motion (Duffy et al., 2011; Manning, 2007; Olszewski, 2008).

\section{Corresponding author:}

Maria Törnqvist, Department of Sociology, Uppsala University, Box 624, Uppsala 75I 26, Sweden.

Email: maria.tornqvist@soc.uu.se 
Couple dancing, like jitterbug, the empirical prism of the present article, has been studied as an involvement of two parties at a time, interacting through the touching of arms, chests, hips and legs, all merging and orienting toward each other to create a shared bodily rhythm of closeness and disruption. Moreover, the interaction with a partner is researched not only through the connection and disconnection of body parts, but also through the olfactory and auditory senses (Duffy et al., 2011). Somewhat neglected, yet vital to understanding the dynamics of the dance floor as well as the emergence of intimacy, is vision. The cheerful and quick jitterbug, a dance characterized by swings performed at some physical distance (couples move while holding one or both hands), requires rather skillful use of vision. In fact, the silent play of eyes is a prerequisite for coordinated movement, as well as for connecting with a partner and establishing emotional presence. Feelings of all sorts - joy, playfulness, flirtation and gravity - interact and are fueled by the gaze within the couple.

Foregrounding vision is a way of expanding the study of dancing to include not only the dynamics of the dance couple, but also their surroundings: other dancers on the floor as well as the observers. Bystanders may be analyzed as a backdrop for the real performance, the staged drama that is in need of "symbolic validation" (Alexander, 2006). However, when unpacking this validation process through the lens of vision and intimacy, the eye does not only symbolize distance and difference, but also connectedness. For example, Reason and Reynolds (2010: X) investigate the "kinesthetic empathy" of audience responses to dance performances, stating that "kinesthesia can be considered as integral to perception, seen as not purely visual but active and multisensory." Thus, the role of the audience should not only be understood in terms of voyeurism from a distance, on the contrary, the eying exchange is itself an active part of dance intimacy.

Analyzing ethnographic and interview data from the dancing festival in Malung, Sweden, we develop a phenomenological approach that is analytically divided in two themes: one addressing vision as a body technique central to couple dancing, the other exploring the use and meaning of vision across spatial distance, such as between dancers and bystanders. Throughout these discussions, we explore how vision is entwined in body movements and emotive flows. Dancing, it is argued, is truly a multi-sensorial experience that highlights the role of the bodily complex in social life (Atkinson and Duffy, 2019: 22). Using the trope of a "sensing eye," addressing multi-sensoriality and visual connectedness in jitterbug, the article probes relations within and beyond that of the dancing couple and shows how the dancing body emerges in and produces not only movement and connection, but also spatiotemporal structures. Foregrounding vision, the study sheds light on an important dimension of how bodies interconnect in and across spatial distance and thus contributes not only to dance studies but also to research on intimacy and body-space sensitivity (see Holmberg, 2019 on dog walking). It adds to the work of body scholars who point to the importance of addressing "what the body does" (Crossley, 1995: 43), particularly when dancing (Foultier, 2013) and, more generally, to phenomenological explorations of embodied encounters with(in) the world. 


\section{Sensing eye}

The framework used to theorize the role of vision in dancing involves three dimensions. First, we stress the importance of (re)embodying vision and attending to it in dance studies, where tactile interaction is often emphasized. Second, the framework foregrounds the fleshy embeddedness of the gaze. The "sensing eye" is situated not only "in me," within the multi-sensorial body, but also in interaction with other bodies, shaping connection and disconnection, reflecting and resisting social hierarchies. Third, the framework pushes the notion of emplacement. The "sensing eye" entails a spatial orientation that is central to social dancing. In the following, these dimensions will be introduced and later on put into play as we explore the world of jitterbug.

First, as stated above, the present article investigates intimate meanings connected to non-verbal aspects of the body, particularly the role of vision. Vision is historically connected with the intellect. In regard to knowledge production, even Greek philosophy considered "vision ... superior to the other senses" (Grosz, 1994: 97). Everyday expressions such as "I see what you mean" indicate a symbolic intertwining of the eye and the mind. And whereas odor, taste, and touch are imagined as being played out on the skin, vision is often depicted as the least bodily sense. Moreover, in the cultural imaginary, sensorial impressions are gendered. Vision is the more rational one and, thus, coded as masculine, while touch is connected to care and femininity (Haraway, 1989). One could say that the specific visual qualities implied by the term "sensing eye" have been forced into the modern division and hierarchization between the abstract mind and the profane body. The primacy of masculine vision and Western ocularcentrism comes at the cost of the de-embodying of sight (Biernoff, 2005: 39-40). Dance and body scholars sometimes make a similar but twisted interpretation: Iris Marion Young, for instance, frames dancing as a "paradigmatic" lived experience "where the 'I' shifts from the eyes to the region of the trunk" (Young, 2005: 52). Dancing is then primarily about the body, not the mind.

Our study explores a bodily culture in which "trunk and eyes" are intertwined in defining the dancing I. Dancing through glancing is part of our multi-sensorial experience of the world. Michel Serres (2016), with his "topology of senses," highlights how sensing is a matter of engaging with a multitude of impressions, thus implying that bodily perceptions mediate each other through an intersensorial process. However, we do not encounter the world through all the senses in equal proportions at any given moment (Pink, 2011: 266). The quality of impressions shifts with time and place and intermingles with personal experiences (Serres, 2016). The complexity of these sensuous affordances by necessity calls for a conceptualization of the gaze beyond Cartesian dichotomies.

Second, our approach reflects the bodily embeddedness of vision and the use of visual perception for relating and connecting. By means of bodily intentionality, we incorporate and "envelop, palpate, espouse the visible things" with our eyes (Merleau-Ponty, 1968: 133), thus involving a kinetic dimension - touch at a distance. ${ }^{1}$ When our eyes land on a 
person or a landscape, they become part of an embodied perception that connects us visually with the world. Eva Hayward uses the trope of "fingeryeyes," accounting for the ways in which cup corals and scientists at a marine lab encounter each other, where perceptions are generated by the encounters themselves when "sensuous impressions" are made (Hayward, 2010: 581). Transferred into the field of jitterbug, we use a reversed trope, addressing the role of vision in terms of a "sensing eye," unfolding how vision and touch intermingle and become vital to movement and connection. This suggests an intricate relationship between esthetics - "the body as seen"-and the connecting practices between rhythmically moving bodies (Atkinson and Duffy, 2019: 22).

However, the permeability between the world and the body implies not only voluntary closeness, but also potential intrusion. Visual recognition can most certainly be unwanted, for instance, in the form of racist, sexist or sexualized gazes. As pointed out by Donna Haraway (1988), the embodiment of vision means that both the viewer and the viewed body do not exist in a vacuum. There are no neutral positions from which the world can be conceived. Although we may take our eyes off someone, being the object of others' sight puts us in an exposed position.

Third, embodied gaze is also essential in creating knowledge about a particular place, like the dance floor, in a "sensuous epistemology of environments" (Feld, 2005). In phenomenological terms, places enter us, we bring them into our bodies. Following Merleau-Ponty, "we must not say that our body is in space, nor for that matter in time. It inhabits space and time" (1945/2014: 140). Departing from the body's own spatiality, he points out that "there would be for me no such thing as space if I did not have a body" (ibid.: 104). Bodies and their corporeal sense-making always take place and, thus, these places are enacted (Ravn, 2017; Sobchack, 2010). Clearly dancing, centered around coordinated movement, offers a relevant case for exploring spatiality. The quality of a particular place - the dance floor with its lightning, noises and crowding bodies - directs sensuous impressions. Mastering the quick jitterbug steps and turns, dancers are asked not only to learn about the spaces they tread, but to embody them, their borders, materials, topology, if they are to navigate the dance well. Dancers must sense the physical environment as a set of "principal distances and directions" that they not only envision, but "'hold in [their] hands"” or "in [their] legs"” (Merleau-Ponty, 1945/2014: 131-132). Combining a visual phenomenology with the framework of emplacement enables an analysis of the sensorial impressions of, or perhaps rather in, the place (Feld and Basso, 1996).

\section{Carnal ethnography}

The present article is based on ethnographic fieldwork, involving participant observation and interviews. In particular, the study draws from fieldwork in the Swedish popular dancing festival in Malung, Dansbandsveckan, an annual, weeklong (re)union of dancers of all levels, ages and geographic origins. This is the peak for all jitterbug and foxtrot enthusiasts across Sweden and neighboring countries, as well as devotees encircling the music connected to the dance scene, the so-called Swedish dansband. The 
festival Dansbandsveckan is visited by around 40,000 people during a period of one week, and its popularity has steadily increased since it first started in 1986. This popularity reflects the long history of this grassroots activity, with a large proportionabout $10 \%$ - of Swedes engaging in open dance nights on a regular basis (Starrin and Steffner Starrin, 2013: 9; see also Larsson and Svensson, 1992). Although both dancing styles are part of the study, the present article primarily addresses jitterbug, in which vision is more significant.

This form of social dancing typically takes place in a vibrant setting with live music. Events vary in size from locally initiated small-scale happenings to large national and international festivals, involving not only dancers but people who come to watch concerts and to enjoy various social aspects of the festivities. The analytical advantage connected to the Malung festival is the access it gives to the dancing culture at a greater intensity and within a social dynamic that is less dependent on already existing relations. Compared to local dance scenes, in which a geographically delimited number of people take classes and engage in social dancing, the multidimensional transient festival-space, in which dancers dance with unfamiliar dancers, also makes vision and eye contact a prominent aspect of seeking contact.

We have carried out fieldwork in Malung at two points in time in 2015 and 2016. On both occasions, we lived in a caravan and indulged in the official festival camping site. The fact that we were total strangers to the culture made it important to draw out the fieldwork in time, allowing us to contrast our very first distanced impressions with the somewhat more embedded ones the following year. Engaging in the everyday life of the festival not only involved taking dance classes and dancing at the six festival venues, but also spending our days with other festival attendants, chitchatting with neighbors and by-passers at the camping site and other festival areas (Figure 1).

These daily non-recorded conversations brought important insights to the fieldwork and made a significant contribution to the formal, open-ended interviews we conducted with 17 experienced dancers of different ages and genders. The interviews were aimed at exploring how dancing is experienced, how the body is used in the dancing communication and how it transmits movement and regulates emotions.

The definition of being a sociologist in the site of observation foremost means doing a great deal of glancing - participant observation is a vision-centered method. Instead of thinking of this as a practice that positions us at a distance from the actual scope, glancing proved to bridge the two practices. Just like other dancers standing alongside the fence, the observing position allowed us to explore the many layers of activities and emotions embedded in and through vision. In fact, our focus on visual experiences partly emanated from our position as beginners. The unskillfulness and lack of knowledge that imbues the newcomer position is not only a deficit, but it also generates productive paths into a culture through "initiation into a bodily craft" (Wacquant, 2004: vii). Approaching couple dancing through the apprentice position, a process of gaining access through bodily transformation made us sensitive to the role of vision in incorporating dancing techniques and regulating symbolic borders. Like 


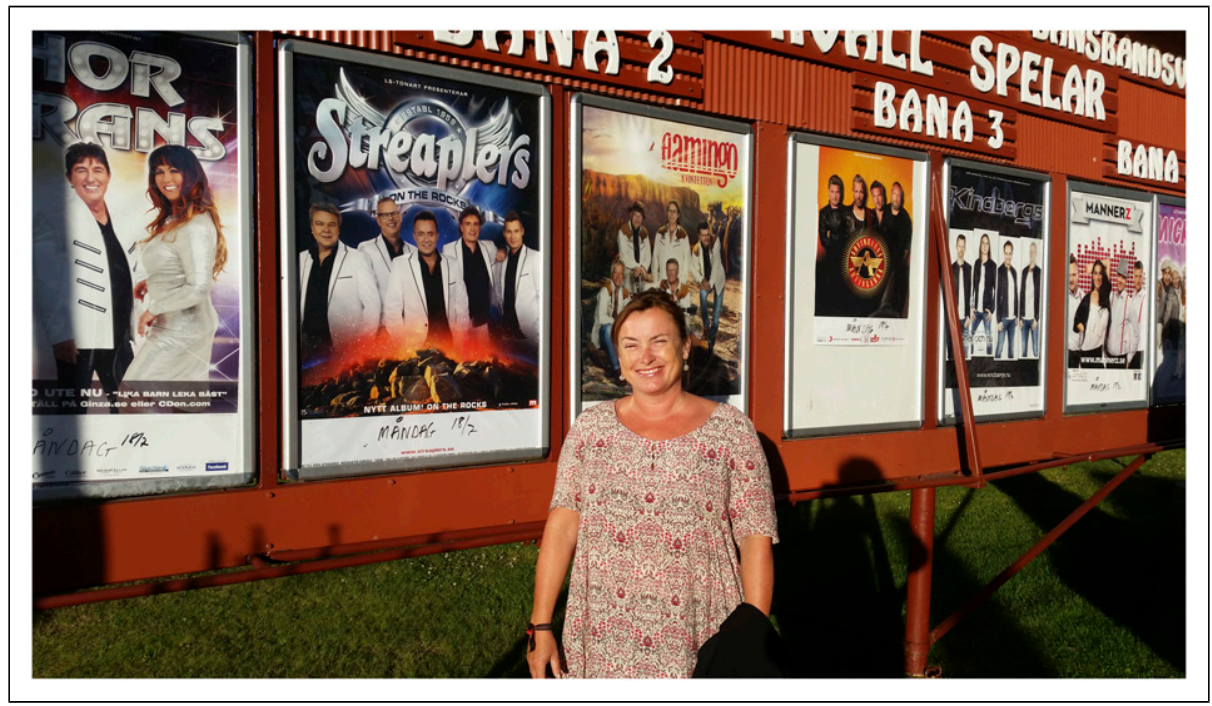

Figure I. On site. Photo by Author.

other beginners, we learned to dance, not only through regular dance classes, but moreover by watching at a distance, by registering how experienced dancers combined steps and rhythm, and by incorporating others' movements into our own bodies, through vision. Taking dance classes during the day and putting our dancing to the test at the festival site at night, we were initiated, in a bodily fashion, into the fine art of dancing through glancing.

Entering the analysis through our own carnal sensations along with other participants' narratives, collected in interviews and observations, is our way of "knowing the human body" while "living it" (Merleau-Ponty, 1945/2014: 205). The outlook of the lived experience of the dancing body is thus a way of forming what Nick Crossley calls a "carnal sociology of the body" (1995: 43; Sobchack, 2010). This carnal methodology requires a reflection on the researcher and how she interacts with the world she studies. Being middle-class academics, women and feminists, there were sometimes experiences of tension. The gendered dimension, for instance, created some predicaments in that the popular dancing events at the festival involve a heterosexual interplay and often display a rather traditional gender order. While doing fieldwork, we were sometimes positioned as objects of flirting, and sometimes "played along" as a tool to gaining knowledge. But instead of approaching matters of class, gender, sexuality or commercialization from the onset, our analytical strategy was to primarily focus on aspects that the dancers themselves indicated were vital to the culture.

The individual fieldnotes that appear throughout the following empirical sections are based on "raw" transcripts, carved out in the midst of the heat, and later on processed in a collective writing exercise aimed at reinstalling embodied memories by engaging with, 
and writing through, the notes. To underline their quality as processed data, notes are placed in squares. Excerpts from interviews, the other type of data, are taken from verbatim transcriptions and appear in the text body.

\section{Dancing through glancing: the couple}

In jitterbug, vision is part of the refined bodily dance techniques. The way jitterbug is led entails kinesthetic touch, mostly using hands, while gaze is an important mediating tool that helps in communicating movement and direction. In addition, the way dancers look transmits and creates emotional energy in the dancing. One prerequisite for "connected dancing" is not only being able to move and affect the other body by touch, but also making one's own body available to the becoming of others through the interplay of vision. As Hayward notes, "perceptions are moved (affected) by the movements and actions that they provoke in other organisms" (2010: 580). In order for moments of "flow" to emerge, dancers must allow themselves to be moved and affected by the look of their partner.

In contrast to, for example, tango, where dancers at times float across the dance floor with eyes closed as a way of intensifying the emotional attendance (Törnqvist, 2013, 2018), jitterbug demands a clear visual intake. In fact, eying is a prerequisite for all sorts of feelings to emerge and a condition for what is sometimes called "dance presence." Communicating eye-to-eye includes a spectrum of expressions, such as subtle blinks, or looking elsewhere. These signals are what experienced dancers read and react to. Some dancers speak of locking vision, meaning closely calibrating and fixating each other by the gaze. The looking of vision causes the eyes of the dancers in a couple to become one common vision for three minutes, the goal being to create an enclosed "dance bubble." Others refer to how a rhythm of closeness and distance is created through playful eye conversations, hooking and looking, on and off. No matter the shade of the feeling, visual connection is central to establishing the couple.

In fact, the gaze is a rather sophisticated communicative tool that can be used both to invite a partner to come closer and to put up borders without involving more potentially offensive expressions, such as removing a hand or verbally telling someone off. In contrast to strong hands that can tie a dance partner's body in an intimate grip, communication through gaze enables quick disconnection. There is always the possibility of looking away. The first step of escaping an intrusive embrace is to look elsewhere, thereby taking part of yourself away from a threatening situation, and of course, it is also a way of searching for escape routes. This visual strategy is primarily used by female dancers. However, disconnecting visually can be experienced as socially difficult, both by the "abandoned" and the "abandoner." Especially for beginner dancers, it may feel "rude" or "greenish" to look away, whereas more experienced dancers are more comfortable both connecting and disconnecting through vision.

In order to be danceable, not only for new dance partners in their scrutinizing search between songs, but to actually be able to synchronize movements and beat, dancers must make themselves visibly present. This experience can be both painfully intimidating and the key to making a wordless bodily connection into a sophisticated communication. 
The music is energetic, 150 beats per minute. The rhythmic movements of the dancers around us signal energy. The positive, joyful feeling transmitted by the sound pulsating from large speakers on the side of the stage is almost contagious and creates a cheerful, inclusive atmosphere. When looking at my partner, I start smiling and move my feet to match the asymmetric basic steps, one foot repeatedly moving forward and backward, while the other stays grounded. The surrounding couples move counterclockwise, forming a vibrant circle of sweaty bodies. My partner has to make sure our frequent spins do not cause us to bump into them. While I spin around and around and around, my legs in concert with the beat, I catch my partner's gaze and a strange sense of trust occurs. Hooking on to that gaze, looking this stranger right in the eye, putting faith in an unknown dance partner, is in a way more intimate than holding hands, or touching the bare skin of an arm. For a moment, I become self-aware. I feel exposed and vulnerable, abruptly thrown out of my comfort zone. When the music shifts, I embrace my uneasy shyness, and the intense, intra-active gaze, with all the emotions that come with it, strikes me to be essential to the bliss of dancing. I stop thinking and just go with the flow.

Eye contact is both nerve-racking and consoling when the apprentice dancer seeks a comforting foundation in an uneasy situation. For a moment, the ethnographer is experiencing the intersubjectivity of the dance and how the gaze of the other becomes blissful. This experience reduces the intrusive closeness that at first creates feelings of being "exposed and vulnerable," completely in the hand of the leader. In fact, and quite to the contrary, the leader signals "you can trust me" by seeking eye contact and keeping it. But to actually "trust," meaning to give up control and let go of selfawareness, does not necessarily require faith in the other, but self-reliance in the form of an embodied dance confidence. The fieldnotes demonstrate that dance intimacy implies power relations and builds on a visual game played under different conditions by dancers at different levels (experienced/beginner) and with shifting positions (leader/follower).

The gaze is an intimate tool of power even when it expresses indifference. In fact, the importance of visual connectedness becomes acutely apparent during instances of disconnection. When Liselott, a semi-professional dancer who participates in dance competitions and works as a dance teacher, is asked to describe situations when "dancing is not working," she refers to the absence of eye contact.

Well, the first thing I come to think about is someone I danced with and he didn't look at me, not once, during the song. It was like, he danced and I ... 'Hello!' ... like 'Should we stop now or are you leading,' he seemed to already be in the next [dance], like 'Okay, should we dance then' and then I 'But what!' It felt like, well, he could have been dancing on his own, so I just had to bear it until the end of the song.

To Liselott, eye contact is vital, not only to have pleasurable dances but moreover for the presence and motivation. The absence of connected vision generates feelings of 
abandonment, which drains energy from the movements. In that absence, the intimacy transmitted by kinesthetic touch, by holding hands and arms and attuning the movement of feet and hips to another dancer, also fades. This relates to the status play of the dance floor. Looking away communicates disinterest or dislike and reveals these feelings in front of a crowded floor. Thus, vision is not only a device for managing and communicating dance steps, it also regulates hierarchies and the social play. Through looking, the fine line between recognition and disregard, friendship and flirting, is negotiated and synchronized.

The fact that vision is both a sensory modality connected with strong feelings - shame, shyness, intimacy, and abandon - and at the same time volatile renders it a complex bodily technique. Just as modern art requires a refined gaze for revealing the many layers in a painting, couple dancing requires an adapted use of vision, played out as a finegrained means of communication, often more subtle than both words and touch. In addition, the multi-sensoriality present in dancing, and the fact that the senses sometimes communicate rapidly without conscious reflection, causes feelings to "leak" through our way of looking. Managing the play of the gaze thus requires practice. With the words of Drew Leder, incorporating body skills requires that we "act to the skill qua thematized goal" (Leder, 1990: 32) and thus make movements and spatiality part of our body structure in ways that allow us to "act from the skill towards something else" (Ravn, 2017: 59; Parvianinen, 2002).

Early on, during beginner classes, newcomers are trained in the fine art of glancing and dancing - a training that challenges visual comfort zones.

At the dance course, I am fumbling with coordination of legs and arms, painfully aware of my unskilled moves. I cannot help looking at my feet, although I'm immediately reprimanded by the instructor. Eventually, when lifting my focus and fixing my eyes somewhere close to my partner's nose, a feeling of embarrassment comes over me.

The teacher tells us to change partners. At short intervals, every three minutes, which is the duration of a dance, the body in front of me changes. The constant exchange makes me aware not only of different dancing styles and levels of perfection, but I come to realize that some are more fun to dance with because of their playful way of making eye contact. A competent guy with a dodging gaze makes me lose focus and interest. The leader with a bubbly, cheeky way of seeking my attention invokes confidence as well as big smiles.

For beginners like me, the meaning of and distinctions between different styles of visioning are still vague. The bodily dance schema is not embedded enough to navigate me through the nuances. At times, I realize that my way of looking creates unwanted feelings. Responding to a song from my childhood, I loosen up my stiffness and let a smile spread on my face, which my partner interprets as an invitation to more intense eye contact. Through my incompetence, I have generated an unpleasant closeness I did not expect or seek. These situations make me strikingly aware, not only of what I look at, but of how I look. At times, self-awareness becomes a hinder to my dancing. My hesitant eyes irritate more experienced dancers. $\mathrm{He}$ doesn't even bother to hide his relief when the music stops and he can turn to a new partner. 
The fieldnotes reveal the difficulties of learning to manage visual communication and how the gaze transmits feelings in ways that may both hinder and enable contact with a partner. If I look at my feet, my partner will disengage. If I look too intensely, she or he might interpret it as an invitation to make closer contact. The reason why visual competence is difficult to both teach and learn, often harder than steps and dance figures, is because vision is embodied. Just like sound or smell, vision is partly placed outside of conscious reflection. With or without our will, the world enters into us, our eyes are seeking it, constantly positioned on something, a landscape, an object or a dance partner. Through physiologic necessity, vision is naturalized to the point that we are rarely aware that we are looking or at what are we looking. Thus, bringing reflection and self-awareness to vision brings a distanced impression in regard to one's own body. In addition, the social rules connected to jitterbug ("look at your partner!") clash with costumes surrounding how one should relate to strangers in other situations, in order to avoid, not enhance, intrusive intimacy.

Controlling when to let a partner in and when to respond distantly within the frame of the visually correct is difficult even for experienced dancers. These dancers as well say that involuntary emotions easily "leak" through their eyes. Moreover, when steps "go wrong," it happens that mistrust and frustration slip out as a negative glance at a partner. Additionally, it is not at all easy to maintain one's visual focus and not transmit involuntary disinterest by looking away from a partner. In the following interview extract, Samantha and Michael, a couple both on and off the dance floor, discuss the importance and the difficulties of keeping eye contact.

M: Well, yes, I like to watch, it feels weird to dance with a girl who looks away. Like, what the hell? Are you here with me or not? I really want to watch and see the eyes - to have eye contact.

S: No, I'm like sloppy, mm, I look a bit here and there, to see what others are doing and so.

M: Yeah well, when you and I perhaps, when we ... I also think it's good that you watch me when we dance and I think that I watch you when we dance, but at the same time, somehow, I must check out what's going on around me ...

This quote expresses a fear of abandon, but also of control, on Michael's part. Mirroring the quite traditional gender order of the jitterbug culture, which mostly consists of male-female couples although there is some variation, Michael's indignation harbors a sense of ownership. "I watch you," comes with a monitoring implication. However, in a gendered analysis, drifting away, like his partner Samantha does, can be interpreted as a marker of integrity. While Michael confirms that when a partner looks away, there is "no one there" to dance with, he admits that it is difficult to stay visually attentive. Looking elsewhere is not always part of a visual strategy for handling emotions; it may also be a way of handling a crowded dance floor or simply a casual way of connecting with the broader encircling culture. Regardless of whether "visual drifting" is a sign of nervousness, a way of 
experiencing other dancers on the floor, disconnecting from a partner or simply a result of people and things entering the visual horizon, it shows that the gaze is deceitful. In fact, intimacy in jitterbug depends on the volatility of the eye, capable both of rapidly enhancing flow and of extinguishing it.

The fact that vision is both a sensory modality connected with strong feelings - shame, shyness, intimacy, abandon, and control — and at the same time part of a non-reflexive bodily situation in which the world enters into us regardless of our intention makes it a complex bodily technique. Mastering the visual aspects of dancing can only be done through repetition and creating new body memory. As Merleau-Ponty frames it, "habit resides neither in thought nor in the objective body, but rather in the body as the mediator of a world" (1945/2014: 146). In accordance with his notion of a "body schema," which guides our way of walking, standing, eating, and talking without reflecting over the movements entailed (1968: 76), the volatile eye must be mastered in accordance with the culture so as not to disturb the dancing. However, not only does the world enter the bodies, the sensing eye also reaches out and draws us into the world. As will become clearer, the emplaced intersubjectivity of the dancers also transfers to others on and through the dance floor.

\section{Visual intersubjectivity: the audience}

Thus far, we have dwelled on the eye-to-eye connection, and disconnection, between leader and follower. Now we will move to the outskirts of the dance floor, to the surrounding circle of bystanders. As previously discussed, it is possible to disconnect from a "boring" dance or a "dodgy" partner by looking elsewhere. The fact that we can move ourselves outside our situated bodies through vision also enables intimate conversations across spatial distance. This is significant in the visual connection between dancers and bystanders (Figure 2).

Instead of being passive contemplators, bystanders' ways of looking are a dynamic part of what is happening on the dance floor (Barbour and Hitchmough, 2014). Just as in the visioning between dancers in a couple, the audience and the dancers connect intraactively, through gaze, in ways that affect the movements on the dance floor. Some couples actively call for audience attention and appear to be drawn toward the outer circle. Advanced dancers seek confirmation for their skills, some actively showing off in front of the encircling crowd; beginners, on the other hand, are often more ambivalent, feeling both pride and shame in the eyes of more advanced dancers who look at them sideways. Thus, the eyes that search through the vibrant body mass are not always welcome.

One informant tells us that although she herself is happy to watch other people dance, she finds other dancers' gazes "awkward." It is not primarily fear of negative evaluation that causes her to dislike the audience's registering eye, rather the fact that the presence of a third party disturbs the dance feeling. "I just want my partner," she says. In other dancers' experience, the onlookers' gazes contain an evaluative power that creates a hierarchical distance and operates in an intrusive and controlling fashion that makes them 


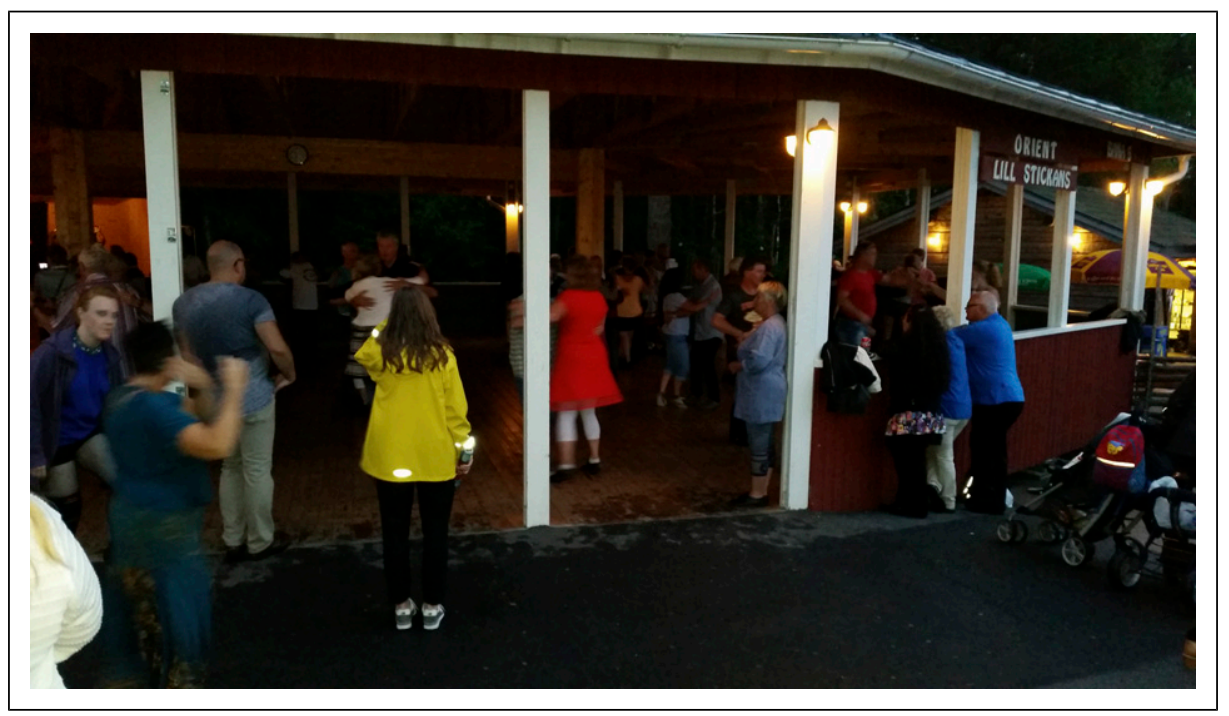

Figure 2. Bystanding. Photo by Author

feel unprotected and vulnerable. Yet standing near the floor, observing the observers, it is striking to register how a hundred people, or more, orient themselves toward the same locus and thus, through visual connection, become part of the radiant dance floor. In Merleau-Ponty's words, it is as "though there were between it [the heart of the visible] and us an intimacy as close as between the sea and the strand" (1968: 130-131). Together with the stream of floating bodies, counterclockwise, the joint movement inwards creates a strange kind of dynamics and a feeling that the place is turning and shrinking.

A ring of spectators encloses the crowded festival floors. Some of them are dancers who stand to the side to rest and have a sip of water, others are looking for the 'right' partner or simply waiting for the dance feeling to kick in. A few are non-dancers, primarily enjoying the live orchestra on stage. Although the reasons for standing on the sideline differ, almost all bystanders turn their attention to the dance floor, making themselves visually attentive to the flow. Especially when skilled dancers pass by the fence, the attention tends to target the same spot, thereby signaling and confirming what is 'worth' looking at. At times, the audience is distant and critical, evaluating bypassing dancers' movements and posture. At other times, their ways of glancing toward the sweaty crowd 'leak' a desire to dance.

I can't take my eyes off those rapidly moving feet, skillfully executing several quick turns in a row. It is my gaze, my way of looking that draws the dance into my body. The smooth and precise way of touching the ground, appearing to be doing a flying exercise, is enthralling. It's no longer my eyes that watch but my feet, they recall the fleeting movement and long to dance. This is how my body learns, not reflexive remembrance but pure muscle memory. 
Particularly for the novice dancer, like the ethnographers in the fieldnotes, bystanding vision is central. We learn to move not only through tactile corporalization, but also through our eyes. Watching others dance and, moreover, engaging in the eye connection within the couple are ways of incorporating movement. Even more advanced dancers use bystanding as a source of inspiration. Stephanie, for instance, a semi-professional dancer who also works as an instructor, talks about the joys of watching others dance. This, she says, is an inspiration that helps develop her own dancing.

Yeah, exactly. I like to move to the music and watch other people while, well, there is so much going on all around as well [...] Yes, I think that's fun. I know for myself; I can stand and enjoy watching others dance and it is amazing how skillful some of them are with their own technique, but also together and that fantastic interaction and then I get so inspired. So I think that I will get that energy when I'm dancing myself too: How did she do that? Why is it flowing like that, can I copy that?

Stephanie perceives embodied observation as a context for trying out new steps and postures. Watching others dance as well as mimicking them is a way of recalling and reinstalling movement into a seemingly passive body. She also accounts for how bystanding causes her to become energized, inspired and moved. In this way, vision creates a channel that transmits energy, flow and presence from dancing bodies to bystanders. Clearly, the object-subject dynamic is more complex than simply defining the motley group of observers as passive.

Visually focusing on moving feet and the experience of incorporating other dancers' movements point to the connections between eye and body, self and other, and passive and active. As expressed both by Stephanie and in the ethnographic account, watching a quick jitterbug can be felt as movement in the bystander's legs and feet. Without actually moving, the observer embodies the movements by "responding" to the solicitation of a visibly attained flow, preparing the torso for the twist necessary to create momentum, making muscles ready to work. Even at a spatial distance, vision connects bodies. Having once incorporated the twists and turns into the corporeal schema makes them recognizable, also when they are attached to another body. Engaged bystanders, like Stephanie, do not primarily experience dancing "objects," but rather how rhythmic movement on the dance floor seamlessly becomes part of their own subjective body, thus transgressing traditional distinctions between active and passive, object and subject.

In the context of couple dancing, however, looking on, instead of being looked at, is also a position imbued with negative feelings like loneliness and frustration. It is the moving bodies that attract attention while the observing position, the glancing, is easily associated with passivity. This is expressed by the wallflower metaphor. 
Literally standing on the side, while everyone else is moving, makes it easy to feel invisible. Facing a floor packed with vibrant energy makes watching appear as the loneliest thing to do. I'm not dressed to just stand. Not engaging in the same energetic movements as the dancers just a few meters away makes me freeze in the chilly Swedish summer evening. When the sun goes down, I put on a cardigan, a thick one. Curling up with myself in the chunky sweater, I feel even more alienated from sweaty dancers with bare arms. In contrast to the heated energy of a hundred moving bodies, I'm almost immobile. Standing next to the crowded floor, watching them melt together under colored spotlights, tuning in to the music in a constant flow, I feel increasingly invisible. Compared to them, I appear to be doing absolutely nothing, stripped of my own body. I'm nothing but eyes, a vision detached from the rest of my body. When my gaze crosses a dancer who holds mine for a second, I become embarrassed and aware of my situatedness as an outsider. Upon realizing my own visibility, as invisible, I adjust to the cultural expectations and although the evening is getting colder, I take off my cardigan. I need to be visible in the right way in order to get to dance.

When the, apparently, radiant crowd enters into the spectator's vision, she too, the observer, becomes visible. The fact that she sees and senses the dancers' presence confirms her existence; [s]'he who sees cannot possess the visible unless [s] he is possessed by it, unless ... [s] [he is one of the visibles' (Merleau-Ponty, 1968: 134-135). The connection between the dance floor and the passive-active audience is a striking example of reversibility. In the reflection above, the ethnographer suddenly experiences the turn of her gaze, how her vision becomes complementary, inhabiting another vision: "myself seen from without ... installed in the midst of the visible" (ibid.: 134). Although these experiences never totally coincide, completing the reversibility, the linkage between seer and the visible marks a connection. Our analysis of bystanders at the festival site implies not only that there is no simple divergence between subject and object, passive and active, but also that the reversibility of vision plausibly implies an intimate connectedness. The dynamics of the dance floor and its surroundings show that vision involves various social interplays, and the notion of a sensing eye reveals a linkage between glancing and dancing - an incorporated intersubjectivity within the broader context of visually connected bodies on and alongside the dance floor.

\section{Conclusion: envisioning touch}

In the introduction, we stated that we are not only interested in the optic dimension of choreography, but in "dancing as vision." In the first empirical section, we specifically addressed visual techniques. The dancers experience vision both as a conscious and a nonreflected part of dancing that in equal measure disturbs and enhances the coordinated movements. Optic engagement can be learned and utilized as a bodily technique, to play and flirt, even with the audience, while smoothly navigating the dance floor. However, as discussed, couple dancing requires adapted use of vision, played out as a fine-grained means of communication, often more subtle than both words and touch. In addition, the 
multi-sensoriality present in dancing, and the fact that senses sometimes communicate rapidly without conscious reflection and regulation, causes feelings to "leak" through dancers' ways of looking.

Investigating the use and meaning of vision in couple dancing pushes, in the second section, the role of bystanders to the fore. The gaze of others may, as with a dance partner, be pleasant and desirable, as well as intrusive and evasive, thus creating feelings of discomfort or even fear and shame. While the observing eye is volatile, deceitful and may hurt, bystanders are also part of a supportive exchange of desire, a position sometimes related to the isolated outsider, who lacks "danceability." This shows that the embodied gaze is far from being only a means of objectification. Rather, dancers who are being looked at may, in certain situations, become active subjects through visual recognition, while those who look can use vision as a channel to access movement and energy. Similar to Atkinson's and Duffy's work on dancing and emplacement, the play of vision in jitterbug stresses the "relationship between visually posited movement- the body as seen - and the rhythmic, affectual, and dynamic potential of the body in motion" (Atkinson and Duffy, 2019: 22).

Our conceptualization of the "sensing eye" in popular dancing thus challenges the lines of division between viewer and viewed and points to intense sensuous intersubjectivity. Looking in and glancing out imply the ever-present spatial component of intersubjectivity. We can cross spatial distances through vision, as our bodies spread out across the dance floor, creating it in concert with the gaze of others. The multiple ways in which we place and move - whether as bystanders, leaders or followers - are intimately tied to our sensorium (Ravn, 2017). The Malung festival site and its dance floors are given shape and come into being through visual sensations. The dance floor is brought into the body through sensuous impressions. We become with the floor, while looking, locking, glancing and dancing.

To sum up, vision is incorporated into our mode of being in and communicating with the world, such that we rarely reflect on what we do when we are looking and how vision affect, and is affected by, social life. Yet the eye is a powerful trope, encompassing meaning related to both love and power. In the present article, we have demonstrated how voluntary and involuntary intimacy in couple dancing depends on vision. While eye contact is part of the emotional life of the dance floor, enabling "dance magic" and "flow," the deceitful and mutable gaze through which intimate flows emerge can also easily extinguish connection. We should, therefore, be careful not to address glancing as a less active and less bodily practice than dancing, but rather see glancing as a way of dancing.

\section{Acknowledgments}

The authors wish to thank the Cultural Matters Group at Uppsala University and the anonymous reviewers for constructive comments. Most of all we thank all participants in the study.

\section{Declaration of conflicting interests}

The author(s) declared no potential conflicts of interest with respect to the research, authorship, and/ or publication of this article. 


\section{Funding}

The author(s) disclosed receipt of the following financial support for the research, authorship, and/or publication of this article: This research was funded by the Swedish Research Council (2014-1465).

\section{ORCID iD}

Maria Tornqvist (D) https://orcid.org/0000-0002-9501-7248

\section{References}

Alexander J (2006) Social Performance. Symbolic Action, Cultural Pragmatics, and Ritual. Cambridge: Cambridge University Press.

Atkinson P and Duffy M (2019) Seeing movement: dancing bodies and the sensuality of place. Emotion, Space and Society 30: 20-26.

Barbour K and Hitchmough A (2014) Experiencing affect through site-specific dance. Emotion, Space and Society 12: 63-72.

Biernoff S (2005) Carnal relations: embodied sight in Merleau-Ponty, Roger Bacon and St Francis. Journal of Visual Culture 4(1): 39-52.

Crespi P (2014) Rhythmanalysis in gymnastics and dance: Rudolf Bode and Rudolf Laban, Body \& Society 20(3-4): 30-50.

Crossley N (1995) Merleau-Ponty, the elusive body and carnal sociology. Body \& Society 1(1): 43-63.

Duffy M, Waitt G., Gorman-Murray A., et al (2011) Bodily rhythms: corporeal capacities to engage with festival spaces. Emotion, Space and Society 4: 17-24.

Feld S and Basso KH (1996) Senses of Place, School of American Research Press.

Feld S (2005) Places sensed, senses placed: toward an sensuous epistemology. In Howes D (ed.) Empire of the Senses. The Sensual Culture Reader. Oxford and New York: Berg. 179-190.

Foultier AP (2013) Towards a phenomenological account of the dancing body: Merleau-Ponty and the corporeal schema. In Foultier AP and Roos C (eds) Material Of Movement and Thought: Reflections on the Dancer's Practice and Corporeality. Stockholm: Fireworks Editions.

Grosz E (1994) Volatile Bodies. Toward a Corporal Feminism. Bloomington: Indiana University Press.

Haraway DJ (1988) Situated knowledges: the science question in feminism and the privilege of partial perspective, Feminist Studies 14(3): 575-599.

Haraway DJ (1989) Primate Visions: Gender, Race, and Nature in the World of Modern Science. New York and London: Routledge.

Hayward E (2010) Fingeryeyes. Impressions of cup corals, Cultural Anthropology, 25(4): 577-599. Holmberg T (2019) Walking, eating, sleeping: Rhythm analysis of human/dog intimacy. Emotion, Space and Society 31: 26-31

Larsson T and Svensson G (1992) Twilight Time. Studier I Svenskt Dansmusikliv. Uppsala: SAMU. Leder D (1990) The Absent Body. Chicago, IL: University of Chicago Press.

Lefevre B (2014) Contemporary dance and its rituals: The example of a work by alain platel. Dancehouse Diary 7: electronic article.

Manning E (2007) Politics of Touch. Sense, Movement, Sovereignty. Minneapolis: University of Minnesota Press. 
Merleau-Ponty M (1945/2014) Phenomenology of Perception. New York: Routledge.

Merleau-Ponty M (1968) The Visible and the Invisible. Evanston: Northwestern University Press.

Olszewski B (2008) El Cuerpo del Baile: the kinetic and social fundaments of Tango. Body \& Society 14(2): 63-81.

Parvianinen J (2002) Bodily knowledge: epistemological reflections on dance. Dance Research Journal 34(1): 11-26.

Pink S (2011) Multimodality, multisensoriality and ethnographic knowing: social semiotics and the phenomenology of perception. Qualitative Research 11(1): 261-276.

Ravn S (2017) Dancing practices: seeing and sensing the moving body, Body \& Society 23(2): $57-82$.

Reason M and Reynolds D (2010) Kinesthesia, empathy, and related pleasures: an inquiry into audience experiences of watching dance. Dance Research Journal 42(2): 49-75.

Serres M (2016) The Five Senses. A Philosophy of Mingled Bodies, London: Bloomsbury Academic.

Sobchack V (2010) Living a 'Phantom Limb': on the phenomenology of bodily integrity, Body \& Society 16(3): 51-67.

Starrin B and Steffner Starrin L (2013) Sverige Dansar. Karlstad: Starrin Förlag.

Törnqvist M (2013) Tourism and the Globalization of Emotions. The Intimate Economy of Tango, New York: Routledge.

Törnqvist M (2018) Rethinking intimacy: Semi-anonymous spaces and transitory attachments in Argentine tango dancing. Current Sociology 66(3): 356-372.

Wacquant LJD (2004) Body \& Soul: Notebooks of an Apprentice Boxer, Oxford: Oxford University Press.

Young IM (2005) On Female Body Experience: "Throwing like a Girl” and Other Essays. Oxford: Oxford University Press.

\section{Author Biographies}

Maria Törnqvist is Associate Professor of Sociology at Uppsala University, Sweden. Her research focuses on intimacy and economies of intimacy, gender politics, and elite education and has appeared in Social Forces, British Journal of Sociology, Sociology, Feminist Review, and Current Sociology. Törnqvist is the author of Tourism and the Globalization of Emotions: The Intimate Economy of Tango (Routledge 2013) and the lyrical essay Merleaus mamma (Ellerström 2020).

Tora Holmberg is Professor in Sociology at Uppsala University, Sweden. Holmberg's cultural sociology approach concerns animal studies, STS (Science and Technology Studies), feminist theory and urban studies, for example, in Urban Animals: Crowding in Zoocities (Routledge, 2015). She has co-edited several books, most recently Death Matters: Cultural Sociology of Mortal Life (Palgrave, 2019). 